\title{
Early Detection of Brain Pathology Suggestive of Early AD Using Objective Evaluation of FDG-PET Scans
}

\author{
James C. Patterson II, ${ }^{1,2,3}$ David L. Lilien, ${ }^{2,4}$ Amol Takalkar, ${ }^{2,4}$ and James B. Pinkston ${ }^{5}$ \\ ${ }^{1}$ Department of Psychiatry, Louisiana State University Health Sciences Center, 1501 Kings Highway, Shreveport, LA 71103, USA \\ ${ }^{2}$ Department of Pharmacology, Toxicology, and Neuroscience, Louisiana State University Health Sciences Center, LA 71103, USA \\ ${ }^{3}$ PET Imaging Center, Biomedical Research Foundation of Northwest Louisiana, LA 71103, USA \\ ${ }^{4}$ Department of Radiology, Louisiana State University Health Sciences Center, LA 71103, USA \\ ${ }^{5}$ Department of Neurology, Louisiana State University Health Sciences Center, LA 71103, USA
}

Correspondence should be addressed to James C. Patterson II, jpatte@lsuhsc.edu

Received 23 April 2010; Revised 6 July 2010; Accepted 10 August 2010

Academic Editor: James B. Brewer

Copyright ( 2011 James C. Patterson II et al. This is an open access article distributed under the Creative Commons Attribution License, which permits unrestricted use, distribution, and reproduction in any medium, provided the original work is properly cited.

\begin{abstract}
The need for early detection of AD becomes critical as disease-modifying agents near the marketplace. Here, we present results from a study focused on improvement in detection of metabolic deficits related to neurodegenerative changes consistent with possible early AD with statistical evaluation of FDG-PET brain images. We followed 31 subjects at high risk or diagnosed with $\mathrm{MCI} / \mathrm{AD}$ for 3 years. 15 met criteria for diagnosis of MCI, and five met criteria for AD. FDG-PET scans were completed at initiation and termination of the study. PET scans were read clinically and also evaluated objectively using Statistical Parametric Mapping (SPM). Using standard clinical evaluation of the FDG-PET scans, 11 subjects were detected, while 18 were detected using SPM evaluation. These preliminary results indicate that objective analyses may improve detection; however, early detection in at-risk normal subjects remains tentative. Several FDA-approved software packages are available that use objective analyses, thus the capacity exists for wider use of this method for MCI/AD.
\end{abstract}

\section{Introduction}

The need for earlier and more accurate detection of Alzheimer's disease (AD) becomes critical with the much anticipated introduction of disease-modifying agents. The earliest detectable changes in AD may well be in the brain tissue itself, and functional brain imaging provides a noninvasive window directly into the brain's activity. The utilization of $\left[{ }^{18} \mathrm{~F}\right]$-fluorodeoxyglucose (FDG) uptake by brain tissue as measured by positron emission tomography (PET) is a welldocumented method for evaluation of brain function as well as dysfunction. Since the earliest examinations of FDG PET studies of brain metabolism [1], there have been reports of a characteristic pattern of decreased brain metabolic activity that is seen in patients with AD. This pattern is generally distinct from that seen in other types of dementia [2, 3]. Other studies have demonstrated a relationship between the magnitude of cognitive impairment in $\mathrm{AD}$ and the extent of these metabolic deficits $[4,5]$, important in that they demonstrate that progression of neurodegenerative changes is associated with both more cognitive impairment and larger PET metabolic deficits. Other reports indicate the potential to detect brain metabolic changes (in the same distinct pattern seen in patients diagnosed with probable $\mathrm{AD}$ ) across groups of premorbid patients, who have not yet developed $M C I$, before symptoms or objective neuropsychological deficits occur [6-8]. However, detecting the subtle metabolic abnormalities associated with the early neurodegenerative changes that eventually will lead to $\mathrm{AD}$ in individual patients (rather than groups) using objective evaluation of PET remains less commonly reported. Objective analysis of PET brain image data has been available since the origins of PET, but has been chiefly utilized in the research arena. While there are numerous studies that report on PET brain imaging and $\mathrm{AD}$, the numbers of studies that make use of advanced computerized digital image processing tools 
are fewer, and fewer still are the studies which use this technology to examine brain activity at the single-subject level [9] (for review see [10]). While it is important to our understanding of the disease process to examine brain activity in group studies, the use of objective statistical image analysis enables the comparison of one brain image to a group of control brain images at the single-voxel level. This means of assessment is important to the clinician as it can provide information that may have an impact on early clinical diagnosis and management, particularly if neuroprotective pharmaceutical strategies are achieved.

Our study examines the usefulness of objective PET brain image analyses compared to standard (subjective visual) interpretation of FDG-PET brain image data. This study included a range of subjects, from individuals who were at risk but cognitively normal through the early mild cognitive impairment (MCI) stage. Even in the subjects who met criteria for MCI, the average MMSE score was 28 (five patients with probable $\mathrm{AD}$ were included as a reference). We demonstrate that in this early population, 11 patients had changes apparent on the SPM examination consistent with early stages of possible AD, but had PET scans that appeared normal to subjective visual interpretation-the standard of care for reading FDG-PET brain scans today. This standard is reviewed further in the discussion, along with the potential implications of adding objective evaluation to this standard. The case is made for objective FDG-PET scan evaluation to improve the current standard for early detection of metabolic changes consistent with possible AD.

\section{Materials and Methods}

2.1. Subjects. This research was conducted in accordance with the guidelines and the approval of the Institutional Review Board. Subjects were recruited from the community with an emphasis on recruitment of subjects with MCI or those in the at-risk cognitively normal state. All subjects were informed of all study procedures and signed informed consent before involvement in the study. In all, a total of 41 subjects were screened. Of these, 9 failed to meet the inclusion or exclusion criteria, and 32 subjects were enrolled. One subject withdrew due to sudden illness before any data collection. (31 used, 15 male, 16 female), with an age range from 56 to 84 , with a mean age of $71 \pm 8$. This study focused on early detection, specifically with regards to patients with subjective memory loss but still not meeting criteria for MCI. To be enrolled in the study, patients must either meet at least MCI criteria and have no other neuropsychiatric problems, or must both have a first-degree relative with $\mathrm{AD}$ and be APoE4+. Subjective memory loss corroborated by friend or family member was preferred. A diagnosis of Major Depression was not grounds for exclusion, based on the high rate of comorbidity [11]. We also included 5 patients with mild AD. Demographics are shown in Table 1.

2.2. Neuropsychological Testing. Patients were evaluated with periodic neuropsychological testing (NP) a total of five times during the study. All subjects were followed longitudinally for three years throughout the study. The initial diagnosis was determined by neuropsychological scores along with the clinical evaluation.

The criteria for a diagnosis of Mild Cognitive Impairment (MCI) were:

(i) memory complaint with informant corroboration,

(ii) objective memory impairment with NP testing,

(iii) normal general cognitive function,

(iv) intact activities of daily living,

(v) not demented.

These criteria were based upon the American Academy of Neurology's practice parameters for early detection of dementia [12]. Neuropsychological tests used to establish objective cognitive impairment included the Folstein MiniMental State Examination, Clock Drawing Test, Trails A \& B, Boston Naming Task, Rey-Osterrieth Complex Figure Task, and the Hopkins Verbal Learning Task. To establish cognitive impairment, subjects must have deficits on at least two scores. If there was only one measure that was abnormal, it was still counted, but denoted as marginal ( \pm in Table 2$)$. For amnestic MCI, subjects had to have deficits on one test of memory with a score $\geq 2 \mathrm{SD}$ outside the age-adjusted normal range, deficits on one test of memory with a score $\geq 1.5 \mathrm{SD}$ outside of the normal range if accompanied by a score on a nonmemory test $\geq 1 \mathrm{SD}$, or deficits on at least two memory tests with scores at least $\geq 1$ SD outside the ageadjusted normal range. To establish cognitive impairment for nonamnestic MCI, subjects had to have a nonmemory cognitive test with a score $\geq 2 \mathrm{SD}$ outside the normal range, or two or more nonmemory cognitive tests with deficits at least $\geq 1$ SD outside the age-adjusted normal range.

Based on the initial NP test scores, subjects were either diagnosed as amnestic MCI, nonamnestic MCI, or combined type MCI. Four subjects did not initially meet criteria for MCI, but were diagnosed at later time points. In addition, five subjects also met clinical criteria for (and were diagnosed with) mild to moderate AD. Furthermore, 11 subjects did not meet criteria for either MCI or AD. This latter subset had subclinical symptoms and/or were normal but at high risk for development of $\mathrm{AD}$ based on the criteria above.

2.3. Control Database. The normal control database was collected for general use in comparison with scans from patients referred for PET brain evaluation. This control database was collected with the approval of the Institutional Review Board of the Louisiana State University Health Science Center at Shreveport. All prospectively identified subjects were recruited by advertisement and fully informed of all risks, and they signed consent forms. Only subjects older than 50 that met the criteria $(n=28)$ were used for the current review. For inclusion in the normal control PET database, subjects had to be physically healthy with no neurological or psychiatric illness, have a normal physical exam, have no memory complaints, and have normal results on basic cognitive screening tests (Folstein MiniMental State Exam $\geq 27$, and Clock Drawing Test $=4$ ). 
TABle 1: Demographics.

\begin{tabular}{lcccccc}
\hline Subjects & Gender $(\mathrm{M} / \mathrm{F})$ & Age \pm SD & min age & max age & Education & MMSE \\
\hline Standard dataset & $14 / 14$ & $65.2 \pm 9.0$ & 51 & 82 & $15 \pm 1.73$ & $28.9 \pm 0.96$ \\
Patients & $15 / 16$ & $71.3 \pm 8.3^{*}$ & 56 & 84 & $14.5 \pm 1.98$ & $28.4 \pm 2$ \\
\hline
\end{tabular}

${ }^{*} P=.01$.

TABLE 2: Results for subjects enrolled in the MCI early detection study.

\begin{tabular}{|c|c|c|c|c|c|c|c|c|c|c|c|c|}
\hline Subject & MMSE & SML & Dx & $\mathrm{SPM}_{1}$ & $\mathrm{SPM}_{2}$ & $\mathrm{PET}_{1}$ & $\mathrm{PET}_{2}$ & 1 & 2 & 3 & 4 & 5 \\
\hline S001 & 30 & - & - & - & - & - & - & & & & & \\
\hline S005 & 30 & - & - & - & - & - & - & & & & - & - \\
\hline S006 & 28 & - & - & - & - & - & - & & & & & - \\
\hline S008 & 29 & - & - & - & - & - & - & $\mathrm{n}$ & $\pm \mathrm{n}$ & $\pm \mathrm{n}$ & $\mathrm{c}$ & - \\
\hline S017 & 30 & - & - & - & - & - & - & c & $c$ & & $\pm \mathrm{n}$ & $\mathrm{a}$ \\
\hline S020 & 30 & - & - & - & - & - & - & $\pm \mathrm{n}$ & $\pm \mathrm{n}$ & c & $\pm \mathrm{n}$ & $\pm \mathrm{n}$ \\
\hline S025 & 29 & - & - & - & - & - & - & & $\mathrm{n}$ & & $\pm \mathrm{n}$ & $\mathrm{n}$ \\
\hline S009 & 27 & - & - & \pm & + & - & - & & $\pm \mathrm{n}$ & & & - \\
\hline S002 & 28 & - & - & + & \pm & + & + & & $c$ & $\pm \mathrm{n}$ & $\pm \mathrm{n}$ & $c$ \\
\hline S003 & 27 & - & - & + & + & - & - & $\mathrm{n}$ & $\pm \mathrm{n}$ & & $\mathrm{n}$ & $\pm \mathrm{n}$ \\
\hline S012 & 30 & + & - & + & + & - & + & & & & & \\
\hline S039 & 29 & + & $( \pm \mathrm{nMCI})$ & - & - & - & - & & & $\pm \mathrm{n}$ & & - \\
\hline S015 & 29 & + & nMCI & - & - & - & - & $\mathrm{n}$ & & $\mathrm{a}$ & $\pm \mathrm{n}$ & - \\
\hline S028 & 28 & + & $\mathrm{nMCI}$ & - & - & - & - & $\pm \mathrm{n}$ & $\pm \mathrm{n}$ & $\mathrm{a}$ & $\pm \mathrm{n}$ & - \\
\hline S019 & 30 & + & $\mathrm{nMCI}$ & - & - & - & - & $\mathrm{n}$ & $\pm \mathrm{n}$ & & $\pm \mathrm{n}$ & $\pm \mathrm{n}$ \\
\hline S027 & 28 & + & $\mathrm{cMCI}$ & - & - & - & - & c & $\mathrm{a}$ & c & $\mathrm{c}$ & $\mathrm{a}$ \\
\hline S034 & 29 & + & cMCI & - & - & + & - & c & c & c & $\mathrm{a}$ & \\
\hline S010 & 29 & + & cMCI & - & \pm & + & & c & $\mathrm{a}$ & c & $\mathrm{a}$ & c \\
\hline S014 & 30 & + & (cMCI) & + & + & + & - & & c & & $\pm \mathrm{n}$ & $\pm \mathrm{a}$ \\
\hline S016 & 27 & + & (cMCI) & \pm & + & - & - & & & c & $\pm \mathrm{n}$ & $\pm \mathrm{a}$ \\
\hline S018 & 29 & + & aMCI & \pm & - & - & - & $\pm \mathrm{a}$ & $\mathrm{a}$ & c & $\pm \mathrm{a}$ & - \\
\hline S022 & 30 & + & $\mathrm{cMCI}$ & + & + & - & - & c & $\mathrm{a}$ & $\mathrm{a}$ & c & $\mathrm{a}$ \\
\hline S013 & 28 & + & $( \pm \mathrm{nMCI})$ & + & ++ & - & + & & $\pm \mathrm{n}$ & $\pm \mathrm{n}$ & & $\pm \mathrm{n}$ \\
\hline S023 & 25 & + & cMCI & + & ++ & - & - & c & $c$ & c & c & c \\
\hline S021 & 29 & + & $\mathrm{nMCI}$ & ++ & ++ & - & - & $\mathrm{n}$ & $\pm \mathrm{n}$ & c & c & \\
\hline S032 & 27 & + & cMCI & +++ & - & + & - & c & $\mathrm{c}$ & c & c & - \\
\hline S031 & 22 & + & $\mathrm{AD}$ & +++ & - & + & - & c & c & c & c & c \\
\hline S033 & 25 & + & $\mathrm{AD}$ & +++ & - & + & - & c & c & - & - & - \\
\hline S038 & 25 & + & $\mathrm{AD}$ & +++ & - & + & - & c & - & - & - & - \\
\hline S040 & 25 & + & $\mathrm{AD}$ & +++ & - & + & - & c & c & c & - & - \\
\hline S041 & 26 & + & $\mathrm{AD}$ & +++ & +++ & - & - & c & c & c & c & c \\
\hline
\end{tabular}

Subj: Subject ID, MMSE: Mini-mental State Exam score, SML: subjective memory loss, Dx: Diagnosis, SPM: Consistent with possible MCI/AD based on inspection of SPM statistical map, PET: Consistent with possible MCI/AD based on inspection of PET scan. There were two PET scans obtained for each patient. One at study initiation and one at the end of the three year study. These are represented by the "1" and " 2 " subscripts for SPM and PET, respectively. $\mathrm{NP}$ testing was done at five timepoints during the three year study, and results for these five examinations are shown in the columns on the right ( 1 through 5). NP testing: (blank): normal, a: amnestic, n: nonamnestic, (-): no data.

Extensive neuropsychological testing was not completed as a cost/benefit analysis indicated that the yield would be minimal in a normal population with no subjective memory loss. All had radiologically normal or unremarkable MRI scans. PET scans were also clinically reviewed by the nuclear medicine physicians utilizing subjective criteria and found to be normal or unremarkable. Furthermore, individual PET scans were removed from the control group and then compared to the remainder of the control group in a "jackknife" fashion, and subjects with PET metabolism changes in a pattern suspicious for the potential of developing dementia (deficits in medial temporal, lateral temporal, 
posterior cingulum "cortex", and posterior parietal regions) were excluded. Demographics for the 28 subjects included in the standard are shown in Table 1.

2.4. PET Image Acquisition and Processing. [18F]-fluorodeoxyglucose (FDG) positron emission tomography (PET) scans were completed at the beginning and at the end of the three-year period. All PET scans were acquired and processed in the same way from the same scanner. All patients were informed to stop any medications potentially affecting brain function 24 hours prior to the study, fast for at least 6 hours prior to the study, and were ensured to have a blood glucose level of less than $200 \mathrm{mg} / \mathrm{dL}$ before administering the radiotracer. All patients were injected intravenously with approximately $15 \mathrm{mCi}(555 \mathrm{MBq})$ of ${ }^{18} \mathrm{~F}-\mathrm{FDG}$ in a room with low ambient noise and light while resting comfortably on a bed. PET images of the brain were obtained 60 minutes later on a GE Advance PET scanner (General Electric Company, Milwaukee, WI). Emission images of the brain were obtained for 10 minutes per bed position as a static acquisition in $2 \mathrm{D}$ mode followed by a 2 minute per bed position transmission scan. The images were reconstructed with ordered subset expectation maximization algorithms and segmented attenuation correction. Images were exported in DICOM (NEMA, Rosslyn, VA) format, and an Analyze 7.5 (Mayo Clinic, Rochester, MN) compatible image was generated from the DICOM slices. This image was imported into the Statistical Parametric Mapping software (SPM, version 8, Wellcome Department of Imaging Neuroscience, University College London), where it was spatially normalized into standardized space (using default settings of 12-parameter linear affine and nonlinear basis functions) and filtered with an $8 \mathrm{~mm}$ isotropic Gaussian filter [13].

2.5. PET Image Review and Analysis. The PET brain images obtained from individual patients were reviewed and interpreted in the standard clinical fashion using subjective visual interpretation on the GE Advance workstation by one of two experienced nuclear medicine physicians. They were blinded to the diagnosis and to the results of the objective SPM analysis.

For objective analyses, PET brain images from individual patients were compared to the control database using voxelby-voxel analysis in SPM [14]. Since there is a small but significant difference in the mean age of the control group and the subjects in the study, age was entered as a nuisance covariate in the SPM analyses. SPM generates an $\operatorname{SPM}(\mathrm{T})$ statistic for the entire brain image that is then converted to the normally distributed $Z$-score. This $3 \mathrm{D} Z$-score map is then surface rendered or overlaid on axial slices of a canonical image for viewing. An $\operatorname{SPM}(\mathrm{T})$ score of 2 and spatial extent of 100 constituted the statistical threshold. This threshold is lower than that used in typical statistical evaluations. The justification for this is two-fold. There is an a priori hypothesis inherent in evaluation of patients with memory loss or cognitive decline that deficits in metabolism are expected in certain specific regions. Furthermore, the pattern of hypometabolism, if present and consistent with early possible $\mathrm{AD}$, is of significance itself, at any threshold. The specific pattern of metabolic deficits putatively associated with neurodegenerative changes due to early Alzheimer's disease process is described below and discussed later in the paper.

FDG-PET scans were evaluated using the SPM results in a similar fashion to the clinical readings, except that the $\operatorname{SPM}(Z)$ map using the above threshold was used to look for patterns of decreased metabolism consistent with possible $\mathrm{AD}$, rather than the PET scan per se. Metabolic deficits in posterior parietal, lateral temporal, posterior cingulum, medial temporal, basal nuclear, and/or posterior hippocampal regions present at this threshold were looked for. PET image maps with smaller (less than 250 voxels), less significant $(Z<3)$, and/or isolated ( 2 regions or less) metabolic deficits were considered negative, as these findings may occur by chance at the low threshold used. Image maps with metabolic deficits that were larger than 250 voxels, had $Z$ scores higher than 3 , and/or had two or more regions in the areas being searched were then further evaluated and graded on a score from marginal $( \pm)$ to strongly positive $(+++)$, depending on the spatial extent, $Z$ score, number of regions, and presence of a "dog-leg" pattern. Results are presented in Table 2. Six subjects (denoted by -) did not complete the second PET scan.

\section{Results and Discussion}

Figure 1 shows four example subjects (S017: no diagnosis, negative SPM evaluation, S003: no diagnosis with positive SPM evaluation, S021: MCI with positive SPM evaluation, and S040: AD). There are some features that are common across all three examples with positive findings (and indeed, across most subjects detected with SPM as being consistent with possible early MCI/AD). This is shown most clearly in the patient with $\mathrm{AD}$. This patient has deficits clearly visible on the actual PET scan, which shows up prominently on the SPM $Z$-map, both overlaid on cross sections and are surface rendered. With $\mathrm{AD}$, the pattern of brain metabolism deficits observed on FDG PET brain scans is characteristic and well established, with bilateral posterior parietal and lateral temporal deficits forming the "dog-leg" pattern that is commonly seen $[4,5,15,16]$ and present on this scan as well, more prominently on the left (all images including surface renderings are in radiological orientation). However, all three subjects with positive findings shown (S003, S021, S040) have both posterior temporal and posterior parietal deficits in metabolism. Also in all three subjects, posterior cingulum hypometabolism is shown. Modern techniques involving statistical analyses typically find posterior cingulum cortex metabolic decline $[17,18]$, but it is often difficult to see these on the actual PET scan until later in the disease process. Medial temporal lobe hypometabolism is not uncommonly seen, especially in earlier stages of the disease process, but has been reported to have more variability $[19,20]$. In our present study, we did not examine or consider frontal metabolic deficits. Frontal lobe hypometabolism is not uncommonly found in patients with 
$\mathrm{MCI} / \mathrm{AD}$, but is nonspecific. One previous study found that patients with $\mathrm{MCI}$ that did not convert to $\mathrm{AD}$ had mainly frontal hypometabolism [20]. Furthermore, PET studies of depression can be associated with frontal hypometabolism [21], and depression is commonly found comorbid with $\mathrm{AD}$ [11]. We did not exclude patients with depression from the current study but also did not examine frontal lobe metabolism, specifically because of this factor.

Table 2 presents a summary of the results from this study. Initially, subjects were diagnosed based on clinical presentation and criteria for MCI or AD. Subjects had to meet standard criteria for MCI to be diagnosed (e.g., subjective memory loss) [12]. There were 11 subjects that did not initially meet criteria for either MCI or AD and 15 who did meet these criteria (one marginal). Four of these 15 subjects did not initially meet criteria, but subsequently did meet the criteria for an MCI diagnosis (two marginally so) later in the study (in parentheses in Table 2). Of the 11 subjects who never met MCI criteria, there were seven subjects who had NP deficits but did not have subjective memory loss criteria for MCI and one who had subjective memory loss but never had NP deficits. NP testing was variable throughout the study in 20 of the 31 subjects. These 20 either had time points where NP test scores were normal (either initially or later in the longitudinal study) or had time points that met criteria for a different subtype of cognitive impairment. Of the 15 subjects diagnosed with MCI, 13 had variable NP results over the time points measured. Of the 11 subjects who did not meet $\mathrm{MCI}$ or $\mathrm{AD}$ criteria, four were consistently negative, and seven had variably positive results. There was variability within subjects with regards to both the presence of and the category of neuropsychological deficit found. For instance, subject 016 was normal, then had a combined deficit, after that became nonamnestic, and finaly, amnestic.

These results appear to reveal several factors, particularly evident in individuals with mild or prodromal disease. First, it is very likely that practice effects impacted patient performance across the five assessments, sometimes resulting in "normal" scores or lack of expected reductions in scores in otherwise affected individuals. Furthermore, individual patient variability, including both short-term differences in performance associated with their disease as well as variability attributable to external, nondisease-related factors such as stress or fatigue could have played a role. This may have led to initial misclassification of some subjects, especially because five subjects who met MCI criteria had no PET findings consistent with possible early AD. Furthermore, the presence of NP deficits in those who are at risk but who deny subjective memory loss is not unexpected, as recently published results demonstrate similar findings [22]. Binder and others examined variability in NP testing in healthy adults and found that deficits were common at low thresholds (1SD) and still present at higher thresholds (2SD). Other reports found that subjects diagnosed with MCI were sometimes reclassified as either normal or other MCI subtype at a follow-up visit [23, 24]. Thus, the presence of abnormalities (especially marginal abnormalities) in subjects who are at risk are not unexpected, and the intrasubject variability in NP results is also consistent with the extant literature.
Standard (subjective visual) FDG-PET scan clinical evaluation initially detected nine of the subjects enrolled in the study. Five of these subjects (all diagnosed with $\mathrm{AD}$ ) did not complete the second PET scan. At the second time point, only three of the remaining 25 subjects were detected. While these numbers seem low, the reason for this is that 15 of the subjects were initially preclinical or in fact normal (although four eventually met criteria for MCI). In addition, even subjects diagnosed with MCI had mild disease, as indicated by the MMSE (mean MMSE for patients with MCI was 28.4).

The SPM evaluation initially detected 14 subjects as having changes in brain metabolism consistent with possible early MCI or $\mathrm{AD}$ that and three subjects as borderline (total $=17$ ). Two of these three subjects were read as positive at the second time point, and one was read as negative (no findings). All subjects that were detected initially were also detected on the second PET scan save for this subject (S018). One subject who was initially negative did not complete the second scan, and five subjects with more advanced disease also did not complete the latter time-point scan. The five subjects diagnosed with possible AD had SPM objective evaluations that were all strongly positive and consistent with possible AD. Only one of these five was rescanned and remained strongly positive.

The variability in findings here deserves mention. While it would be expected that subjects with a diagnosis of MCI and/or findings on SPM objective evaluation would progress, not all subjects would. Some subjects progressed, some had no substantial change, and two actually appeared to have SPM findings that were smaller on the second scan. Bozoki and others recently discovered the presence of "cognitive plateaus" in $22 \%$ of the patients with $\mathrm{AD}$ that they studied $(n=243)$ [25]. The average length of the plateau was 3.6 years, with the range being 3 to 7 years. This is important in that this could be a reflection of a "metabolic plateau" where there is a lack of progression in the deficits in hypometabolism and could help explain the lack of change in some subjects in this present study. Another report demonstrated that higher premorbid intellectual function (a measure of cognitive reserve) can impact not just cognitive performance but also FDG-PET brain metabolism [26]. It is thus possible that subjects in the preclinical or very early stages of $\mathrm{AD}$ might have findings on objective PET evaluation, but have little to no subjective and/or objective memory loss.

The diagnosis of $\mathrm{MCI}$ is made based upon the presence of subjective memory loss with objective cognitive impairment on NP testing. In this study, we found that 15 subjects met criteria for a diagnosis of MCI either initially or at a subsequent time point. Of these, six were consistently negative with respect to metabolic deficits on objective SPM evaluation. Thus, there were a subset of subjects with no diagnosis who had SPM findings and a subset of subjects diagnosed with MCI who had no SPM findings.

In summary, there was little that was consistent with respect to the subjects diagnosed with MCI or the at-risk subjects who did not meet criteria. There were some in each category who had no SPM findings and some that did. 

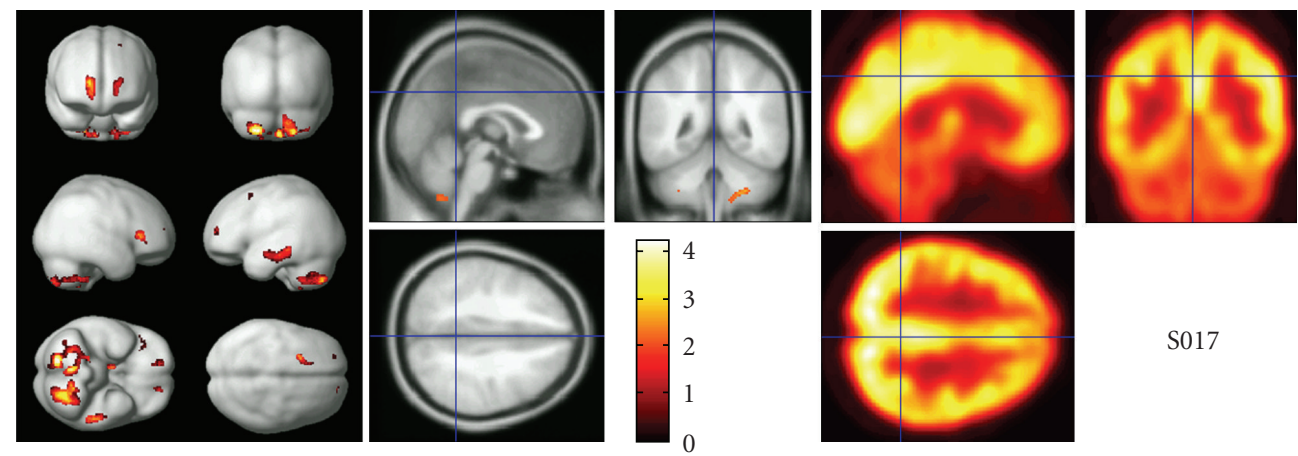

(a)
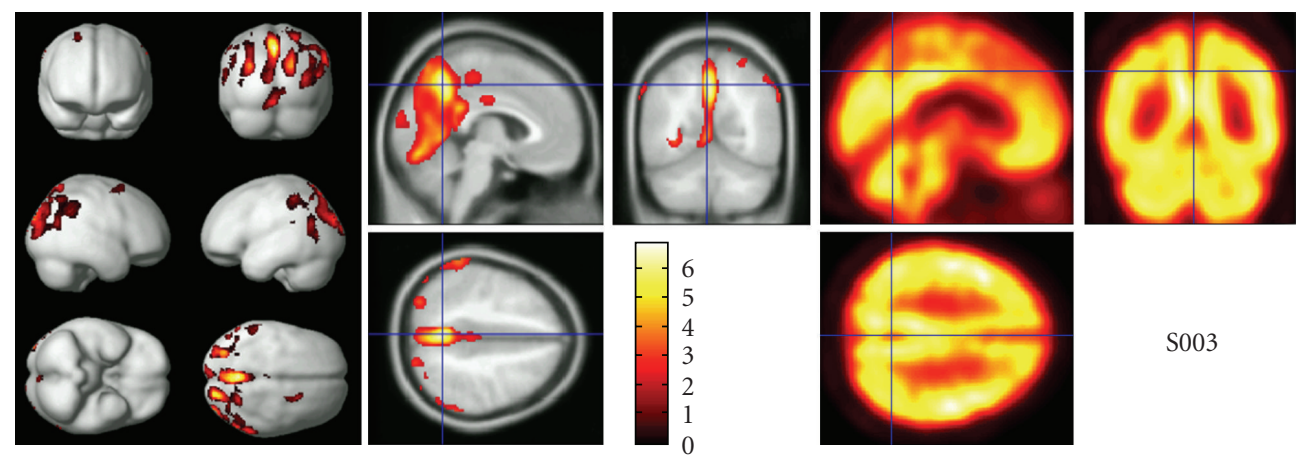

(b)
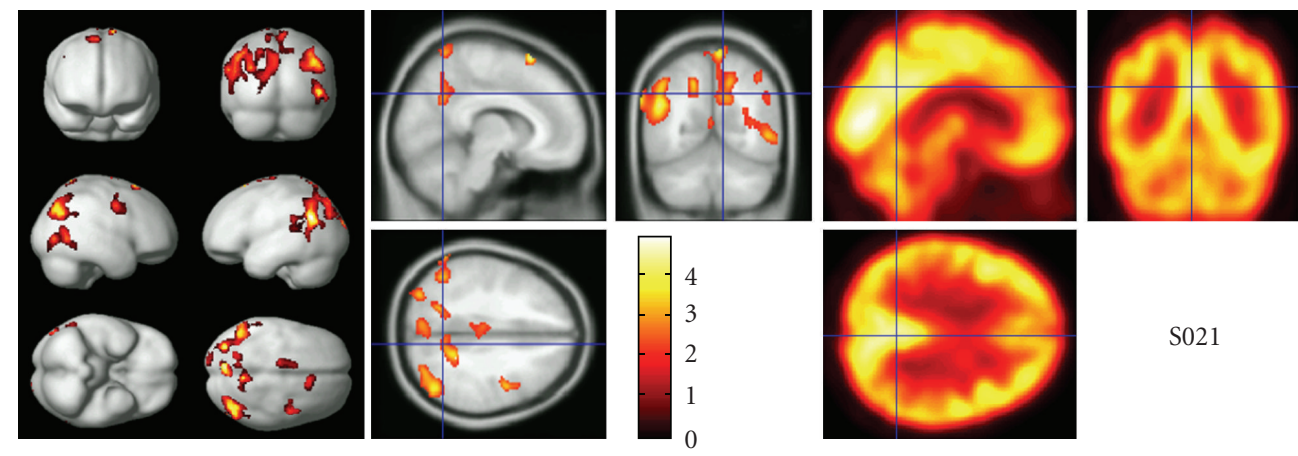

S021

(c)
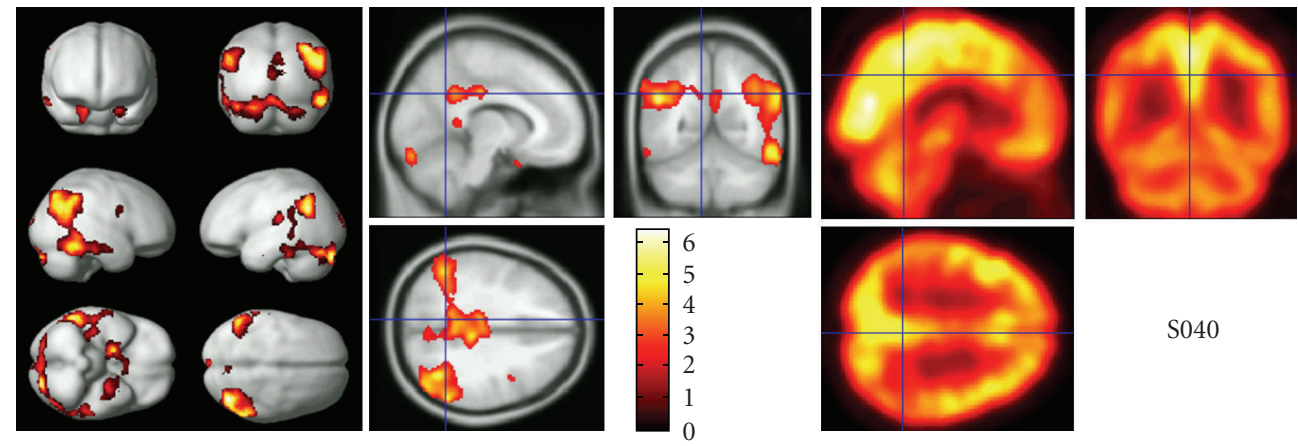

S040

(d)

FIGURE 1: Example results from four subjects in the study. Each row is one subject. The color bar in each row represents the scale for the $Z$-score in that subject. The left column depicts the SPM $Z$-map surface rendering, the middle column depicts the SPM $Z$-map overlaid on a canonical template image in cross section, and the right column shows the FDG-PET scan in cross-section. 
Since memory loss and objective impairment are both requirements for a diagnosis of $\mathrm{MCI}$, there is of course close association here, but even in this subgroup, nine of the $10 \mathrm{MCI}$ subjects did not maintain consistent type of deficit on NP scores.

3.1. Prediction of MCI/AD Diagnosis. The current standard of care for diagnosis of $\mathrm{AD}$ is a diagnosis of exclusion made clinically. The gold standard remains a brain biopsy to look for neurofibrillary tangles and amyloid plaques; however, this is rarely done until autopsy. The use of FDG-PET has been considered for augmentation of the standard of care to improve accuracy and early detection, but cost and accuracy with regard to early detection of MCI were problematic [27]. However, previous evaluations in this respect did not include the use of objective statistical evaluation of FDG-PET scans. Two current large ongoing studies (ADNI: Alzheimer's Disease Neuroimaging Initiative, and NEST-DD: Network for Standardization of Dementia Diagnosis) also support the use of objective statistical image analyses for early detection of $\mathrm{AD}[28]$.

Our research demonstrates a possible advantage with objective SPM evaluation over standard (subjective visual) clinical interpretation in that clinically relevant patterns of hypometabolism were observed in some subjects not yet diagnosed and were not observed in some subjects who were diagnosed with MCI. It is worth mentioning that the former may represent false positive identification and the latter false negatives. While these results presented here are variable and somewhat confusing, it is important to understand the context of a group of subjects consisting mostly of at-risk cognitively normal or very early clinical subjects (save for five patients with $\mathrm{AD}$, included as positive controls). In this population on the borderline of possible development of $\mathrm{AD}$, all three detection algorithms (NP testing, standard visual interpretation, objective SPM interpretation) were variably ineffective. Where there was agreement among diagnosis and all three algorithms, there was clarity. However, whether either subjects with no SPM findings and positive NP findings (and vice-versa) eventually develop AD must be evaluated at future time points, and so this work must remain preliminary. Thus, while the presence of false positives or negatives cannot be determined for certain at this time, it is possible that objective statistical evaluation of FDG-PET brain scans could improve the detection rate for nuclear medicine physicians. While these results must be classified as preliminary (diagnosis of $\mathrm{AD}$ is not definitive-especially in at-risk cognitively normal subjects), it is supported by the extant literature demonstrating FDG PET detection (via objective statistical evaluation) in premorbid subjects, both across groups [6-8] and in other previous studies examining individual subjects [29-32].

The pattern of deficits in metabolism observed in Alzheimer's disease is consistent: posterior cingulate, posterior parietal, lateral temporal, medial temporal/hippocampus, sometimes basal nuclear and variably frontal hypometabolism. There can be variance within this pattern (e.g., some cases are unilateral, some are bilateral) but the regions themselves are highly consistent. Practically, all published articles on FDG-PET imaging of AD spanning 30 years find hypometabolism in these regions (see e.g., $[1-3]$ ). As early as 1995, Minoshima calls this pattern "diagnostic" when referring to the pattern of hypometabolism seen in the parietal and temporal regions of FDG-PET brain scans from patients with $\mathrm{AD}$ [33]. This pattern is a hallmark feature of $\mathrm{AD}$, and other neurodegenerative diseases have diverse patterns of hypometabolism [2, 3, 31] (for review see [32]). With respect to the frontotemporal lobar dementias (FTLD), Pick's disease has a different clinical picture, and the statistical map would demonstrate mostly the frontotemporal pattern of metabolic deficits $[34,35]$. The semantic dementia variant of FTLD may be a potential diagnostic challenge, but the pattern of memory loss is different (semantic versus short term), and the metabolic pattern is typically restricted to the temporal lobes, especially early in the disease process [36]. In $\mathrm{AD}$, deficits in metabolism may appear in medial temporal regions, but they also quickly appear in the posterior cingulum and/or posterior parietal cortices, and typically appear in these latter regions first. Lewy body dementia would be the most problematic differential diagnostic issue, as it has a partially similar pattern of metabolic deficits and a clinical presentation that could be confused. However, the posterior metabolic findings are typically accompanied by a distinctive occipital lobe hypometabolism [2,37, 38]. Vascular dementia has a pattern that depends upon the region of the vascular lesion. It is possible to have vascular lesions and subsequent hypometabolic findings in regions that coincide with those typically seen in $\mathrm{AD}$; however, this would be atypical as patterns of vascularization in humans do not predispose towards infarcts in medial temporal or posterior cingulum regions. In addition, the clinical picture would once again be different.

The standard of care in PET brain imaging for detection of $\mathrm{AD}$ is subjective visual interpretation of the FDG-PET brain scan. The purpose of this paper was to demonstrate an advantage of objective SPM evaluation over the standard clinical (subjective visual) PET brain image evaluation, and in this regard was met with a measured degree of success. A key concept is that objective statistical evaluation of PET brain image data has the potential to significantly extend the nuclear medicine physician's ability to provide pertinent data to the referring physician to improve diagnostic accuracy. Previous evaluations of PET imaging as a standard for detection of $\mathrm{AD}$ determined that PET imaging for early detection was "optional" (reviewed in [27]). However, these previous examinations did not consider the utility of objective statistical analyses of PET brain imaging data; they just considered subjective visual interpretation. This is a critical and overlooked detail, and hopefully this and other studies will provide the impetus for re-evaluation (inclusive of objective analyses) of FDG-PET in early detection of possible $\mathrm{AD}$. As demonstrated here in this study, objective PET evaluation increased the detection rate in patients diagnosed with early MCI from 5/15 to 9/15 subjects (inclusive of both time points). Further, the consistent lack of objective PET findings in the other subjects may be indicative of misdiagnosis with MCI. SPM evaluation also detected changes in four subjects who did not meet criteria for MCI (one was also detected by standard clinical PET evaluation), 
one of whom had no NP deficits, indicating the presence of metabolic changes that could herald the eventual onset of $\mathrm{AD}$ in these at-risk subjects. However, given the uncertainty in diagnosis at this stage of the disease process, more sensitive detection of hypometabolic regions possibly representing very early $\mathrm{AD}$ could be problematic if used in isolation. If used as a tool for the nuclear medicine physician to provide more sensitive answers to referring physicians, it may be of benefit in cases with clinically correlated illness.

\section{Conclusions}

These results indicate that objective statistical analysis of FDG-PET brain images has the potential to detect changes in brain metabolism that appear consistent with the neurodegenerative changes associated with $\mathrm{AD}$ in individual patients diagnosed with MCI as well as in the at-risk cognitively normal state at a higher rate than with standard clinical PET brain scan evaluation alone. This is an important finding, as the need for early detection grows as our ability to treat this neurodegenerative disease improves. Blood tests that screen for early changes in $\mathrm{AD}$ progression may eventually become readily available and sensitive enough for screening purposes; however, Alzheimer's disease is a brain disorder, and thus examining actual brain activity will always be an optimal means of assessing disease state. Given that there are now several FDA approved software packages available [10], objective evaluation of FDG PET brain scans for the early detection of metabolic deficits related to the neurodegenerative changes of Alzheimer's disease in the MCI stage is currently available to most PET imaging centers. These objective software packages are specifically designed to function as tools that improve detection capability and thus enable the nuclear medicine physician to aid the referring physician in early diagnosis and subsequent treatment decisions.

\section{Acknowledgment}

This paper was supported by a grant from the Biomedical Research Foundation of Northwest Louisiana.

\section{References}

[1] R. P. Friedland, T. F. Budinger, and E. Ganz, "Regional cerebral metabolic alterations in dementia of the Alzheimer type: positron emission tomography with [18F]fluorodeoxyglucose," Journal of Computer Assisted Tomography, vol. 7, no. 4, pp. 590-598, 1983.

[2] P. M. Kemp and C. Holmes, "Imaging in dementia with Lewy bodies: a review," Nuclear Medicine Communications, vol. 28, no. 7, pp. 511-519, 2007.

[3] S. Pakrasi and J. T. O'Brien, "Emission tomography in dementia," Nuclear Medicine Communications, vol. 26, no. 3, pp. 189-196, 2005.

[4] R. Duara, C. Grady, and J. Haxby, "Positron emission tomography in Alzheimer's disease," Neurology, vol. 36, no. 7, pp. 879-887, 1986.

[5] J. V. Haxby, C. L. Grady, and R. Duara, "Neocortical metabolic abnormalities precede nonmemory cognitive defects in early
Alzheimer's-type dementia," Archives of Neurology, vol. 43, no. 9, pp. 882-885, 1986.

[6] G. W. Small, L. M. Ercoli, D. H. S. Silverman et al., "Cerebral metabolic and cognitive decline in persons at genetic risk for Alzheimer's disease," Proceedings of the National Academy of Sciences of the United States of America, vol. 97, no. 11, pp. 6037-6042, 2000.

[7] M. J. De Leon, A. Convit, O. T. Wolf et al., "Prediction of cognitive decline in normal elderly subjects with 2[18F]fluoro-2-deoxy-D-glucose/positron-emission tomography (FDG/PET)," Proceedings of the National Academy of Sciences of the United States of America, vol. 98, no. 19, pp. 10966-10971, 2001.

[8] E. M. Reiman, R. J. Caselli, K. Chen, G. E. Alexander, D. Bandy, and J. Frost, "Declining brain activity in cognitively normal apolipoprotein E $\varepsilon 4$ heterozygotes: a foundation for using positron emission tomography to efficiently test treatments to prevent Alzheimer's disease," Proceedings of the National Academy of Sciences of the United States of America, vol. 98, no. 6, pp. 3334-3339, 2001.

[9] L. Mosconi, H. T. Wai, A. Pupi et al., "18F-FDG PET database of longitudinally confirmed healthy elderly individuals improves detection of mild cognitive impairment and Alzheimer's disease," Journal of Nuclear Medicine, vol. 48, no. 7, pp. 1129-1134, 2007.

[10] J. Patterson II, A. Minagar, N. Natarajan, and A. Takalkar, "Alzheimers disease detection with objective statistical evaluation of FDG-PET brain scans: essential methodology for early identification," Future Neurology, vol. 5, no. 2, pp. 259-276, 2010.

[11] G. S. Zubenko, W. N. Zubenko, S. McPherson et al., "A collaborative study of the emergence and clinical features of the major depressive syndrome of Alzheimer's disease," American Journal of Psychiatry, vol. 160, no. 5, pp. 857-866, 2003.

[12] R. C. Petersen, J. C. Stevens, M. Ganguli, E. G. Tangalos, J. L. Cummings, and S. T. DeKosky, "Practice parameter: early detection of dementia: mild cognitive impairment (an evidence-based review)," Neurology, vol. 56, no. 9, pp. 11331142, 2001.

[13] K. J. Friston, J. Ashburner, C. D. Frith, J.-B. Poline, J. D. Heather, and R. S. J. Frackowiak, "Spatial registration and normalization of images," Human Brain Mapping, vol. 3, no. 3, pp. 165-189, 1995.

[14] K. J. Friston, A. P. Holmes, K. J. Worsley, J.-P. Poline, C. D. Frith, and R. S. J. Frackowiak, "Statistical parametric maps in functional imaging: a general linear approach," Human Brain Mapping, vol. 2, no. 4, pp. 189-210, 1994.

[15] B. Hagberg and D. H. Ingvar, "Cognitive reduction in presenile dementia related to regional abnormalities of the cerebral blood flow," British Journal of Psychiatry, vol. 128, no. 3, pp. 209-222, 1976.

[16] R. P. Friedland, T. F. Budinger, E. Koss, and B. A. Ober, "Alzheimer's disease: anterior-posterior and lateral hemispheric alterations in cortical glucose utilization," Neuroscience Letters, vol. 53, no. 3, pp. 235-240, 1985.

[17] S. Minoshima, N. L. Foster, and D. E. Kuhl, "Posterior cingulate cortex in Alzheimer's disease," The Lancet, vol. 344, no. 8926, p. 895, 1994.

[18] S. Minoshima, B. Giordani, S. Berent, K. A. Frey, N. L. Foster, and D. E. Kuhl, "Metabolic reduction in the posterior cingulate cortex in very early Alzheimer's disease," Annals of Neurology, vol. 42, no. 1, pp. 85-94, 1997. 
[19] M. J. de Leon, L. Mosconi, K. Blennow et al., "Imaging and CSF studies in the preclinical diagnosis of Alzheimer's disease," Annals of the New York Academy of Sciences, vol. 1097, pp. 114 145, 2007.

[20] D. Anchisi, B. Borroni, M. Franceschi et al., "Heterogeneity of brain glucose metabolism in mild cognitive impairment and clinical progression to Alzheimer disease," Archives of Neurology, vol. 62, no. 11, pp. 1728-1733, 2005.

[21] T. A. Kimbrell, T. A. Ketter, M. S. George et al., "Regional cerebral glucose utilization in patients with a range of severities of unipolar depression," Biological Psychiatry, vol. 51, no. 3, pp. 237-252, 2002.

[22] L. M. Binder, G. L. Iverson, and B. L. Brooks, "To err is human: "abnormal" neuropsychological scores and variability are common in healthy adults," Archives of Clinical Neuropsychology, vol. 24, no. 1, pp. 31-46, 2009.

[23] A. J. Jak, M. W. Bondi, L. Delano-Wood et al., "Quantification of five neuropsychological approaches to defining mild cognitive impairment," American Journal of Geriatric Psychiatry, vol. 17, no. 5, pp. 368-375, 2009.

[24] E. Teng, K. D. Tingus, P. H. Lu, and J. L. Cummings, "Persistence of neuropsychological testing deficits in mild cognitive impairment," Dementia and Geriatric Cognitive Disorders, vol. 28, no. 2, pp. 168-178, 2009.

[25] A. C. Bozoki, H. An, E. S. Bozoki, and R. J. Little, "The existence of cognitive plateaus in Alzheimer's disease," Alzheimer's and Dementia, vol. 5, no. 6, pp. 470-478, 2009.

[26] G. E. Alexander, M. L. Furey, C. L. Grady et al., "Association of premorbid intellectual function with cerebral metabolism in Alzheimer's disease: implications for the cognitive reserve hypothesis," American Journal of Psychiatry, vol. 154, no. 2, pp. 165-172, 1997.

[27] S. Minoshima, "Imaging Alzheimer's disease: clinical applications," Neuroimaging Clinics of North America, vol. 13, no. 4, pp. 769-780, 2003.

[28] C. Haense, K. Herholz, W. J. Jagust, and W. D. Heiss, "Performance of FDG PET for detection of Alzheimer's disease in two independent multicentre samples (NEST-DD and ADNI)," Dementia and Geriatric Cognitive Disorders, vol. 28, no. 3, pp. 259-266, 2009.

[29] J. H. Burdette, S. Minoshima, T. V. Borght, D. D. Tran, and D. E. Kuhl, "Alzheimer disease: improved visual interpretation of PET images by using three-dimensional stereotaxic surface projections," Radiology, vol. 198, no. 3, pp. 837-843, 1996.

[30] K. Ishii, M. Sasaki, M. Matsui et al., "A diagnostic method for suspected Alzheimer's disease using $\mathrm{H} 215 \mathrm{O}$ positron emission tomography perfusion Z score," Neuroradiology, vol. 42, no. 11, pp. 787-794, 2000.

[31] N. L. Foster, J. L. Heidebrink, C. M. Clark et al., "FDG-PET improves accuracy in distinguishing frontotemporal dementia and Alzheimer's disease," Brain, vol. 130, no. 10, pp. 26162635, 2007.

[32] J. C. Patterson II, D. L. Lilien, A. Takalkar, R. E. Kelley, and A. Minagar, "Potential value of quantitative analysis of cerebral PET in early cognitive decline," American Journal of Alzheimer's Disease and other Dementias, vol. 23, no. 6, pp. 586-592, 2009.

[33] S. Minoshima, K. A. Frey, R. A. Koeppe, N. L. Foster, and D. E. Kuhl, "A diagnostic approach in Alzheimer's disease using three-dimensional stereotactic surface projections of fluorine18-FDG PET," Journal of Nuclear Medicine, vol. 36, no. 7, pp. 1238-1248, 1995.
[34] L. Mosconi, W. H. Tsui, K. Herholz et al., "Multicenter standardized 18F-FDG PET diagnosis of mild cognitive impairment, Alzheimer's disease, and other dementias," Journal of Nuclear Medicine, vol. 49, no. 3, pp. 390-398, 2008.

[35] T. Grimmer, J. Diehl, A. Drzezga, H. Förstl, and A. Kurz, "Region-specific decline of cerebral glucose metabolism in patients with frontotemporal dementia: a prospective $18 \mathrm{~F}-$ FDG-PET study," Dementia and Geriatric Cognitive Disorders, vol. 18, no. 1, pp. 32-36, 2004.

[36] J. Diehl, T. Grimmer, A. Drzezga, M. Riemenschneider, H. Förstl, and A. Kurz, "Cerebral metabolic patterns at early stages of frontotemporal dementia and semantic dementia. A PET study," Neurobiology of Aging, vol. 25, no. 8, pp. 10511056, 2004.

[37] K. Herholz, "PET studies in dementia," Annals of Nuclear Medicine, vol. 17, no. 2, pp. 79-89, 2003.

[38] K. Ishii, T. Soma, A. K. Kono et al., "Comparison of regional brain volume and glucose metabolism between patients with mild dementia with lewy bodies and those with mild Alzheimer's disease," Journal of Nuclear Medicine, vol. 48, no. 5, pp. 704-711, 2007. 


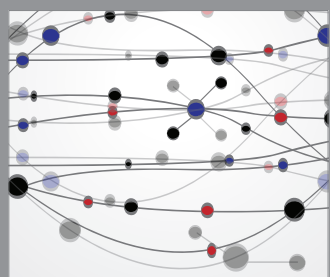

The Scientific World Journal
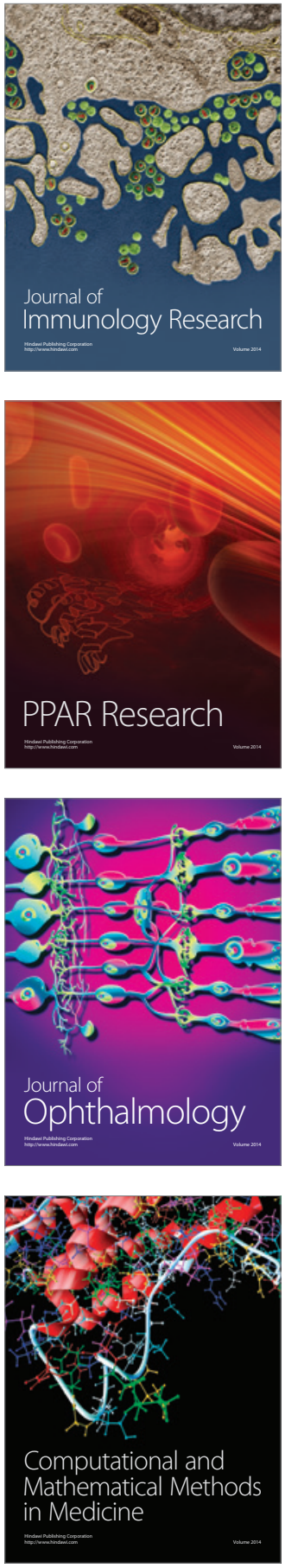

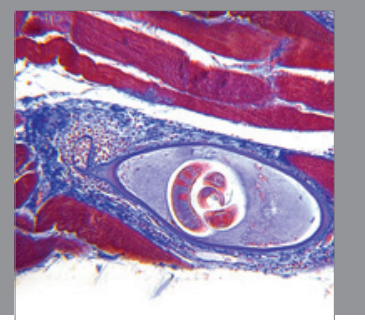

Gastroenterology

Research and Practice
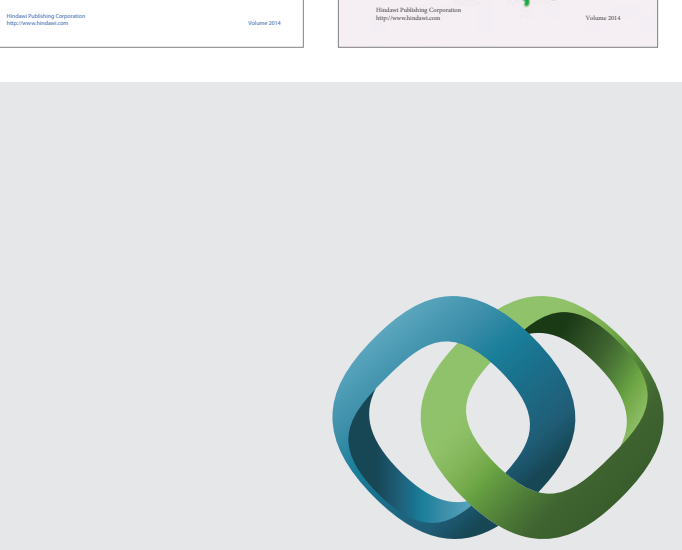

\section{Hindawi}

Submit your manuscripts at

http://www.hindawi.com
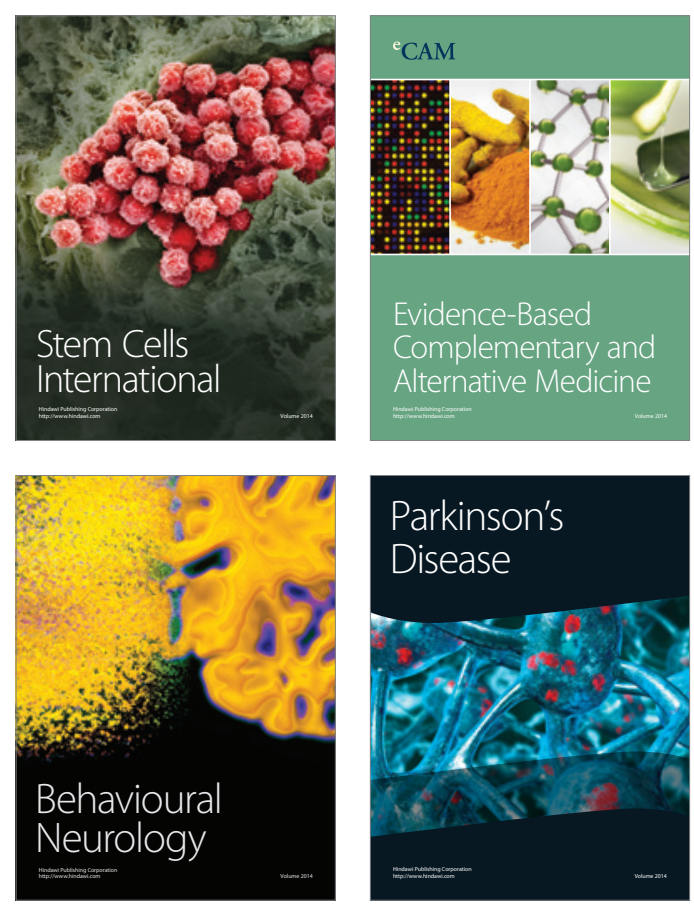

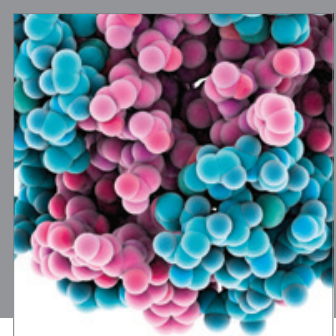

Journal of
Diabetes Research

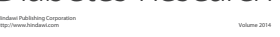

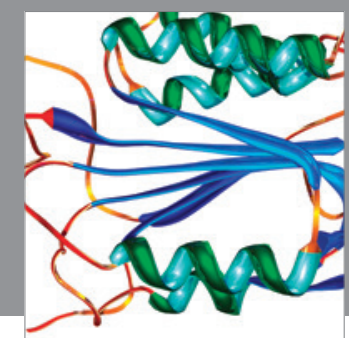

Disease Markers
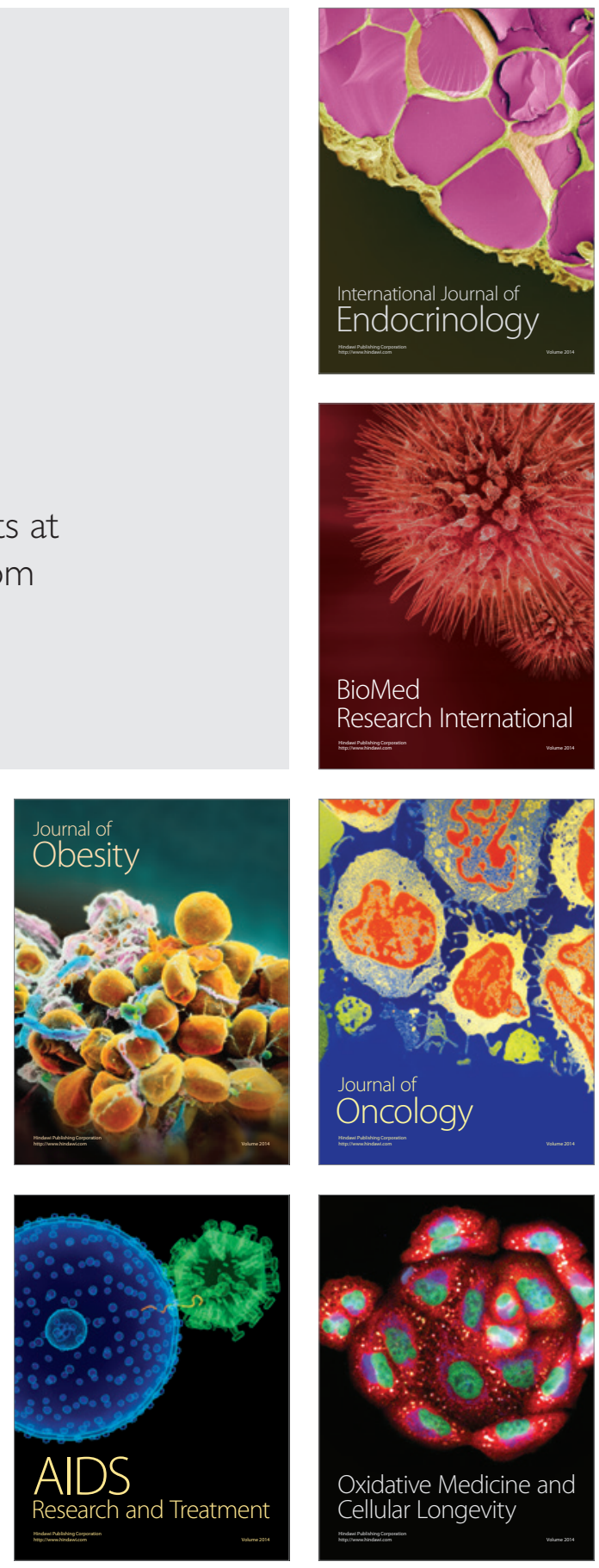